\title{
Plasma P-selectin Predicts Long-term Cardiovascular Events in Hospitalized Patients with Suspected Coronary Artery Disease and Preserved Left Ventricular Function: A 10-Year Follow-Up Study
}

\author{
Wei-Siang Chen, Shih-Jen Chen, Chen-Chin Lee, Wen-Jin Cherng, Min-Hui Liu, Chao-Hung Wang
}

Background: A variety of biomarkers have been investigated on their values to predict cardiovascular outcomes, such as high-sensitivity C-reactive protein (hs-CRP), fibrinogen, troponin-I (TnI), and soluble P-selectin (sP-sel). By a design of head-to-head comparison, this study sought to figure out the long-term prognostic values of these parameters in patients hospitalized with suspected coronary artery disease.

Methods: A total of 170 patients hospitalized with suspected coronary artery disease were enrolled and followed up for an average of 10 years. sP-sel, hs-CRP, TnI, and fibrinogen levels were measured. During the follow-up period, cardiac events were recorded including cardiac death, non-fatal myocardial infarction, and acute coronary syndromes with hospitalization.

Results: $\quad$ For all 170 patients, with a median follow-up time of $9.86 \pm 3.87$ years, no parameter was able to significantly predict the occurrence of cardiac events. In subgroup analysis, an sP-sel of $\geq 63.5 \mathrm{ng} / \mathrm{ml}$ significantly predicted the development of all composite cardiac events only in patients with a left ventricular ejection fraction $>50 \%$ ( $n=94, p=0.04)$. However, the levels of hs-CRP, TnI, and fibrinogen did not have significant predictive values. Multivariate analysis also demonstrated the independent predictive value of sP-sel on all cardiac events (hazard ratio $=5.82, p=0.02$ ). All parameters, including sP-sel, could not demonstrate prognostic values in patients with a left ventricular ejection fraction $\leq 50 \%(n=76)$.

Conclusions: In this 10-year long-term follow-up study, sP-sel was demonstrated to have prognostic values in predicting the cardiac events in patients with preserved left ventricular systolic function.

(Biomed J 2013;36:137-143)

\begin{abstract}
At a Glance Commentary
Scientific background of the subject

A variety of biomarkers have been used for cardiovascular prognostic prediction. By a design of head-to-head comparison, this study sought to figure out the long-term prognostic values of these parameters in patients hospitalized with suspected coronary artery disease.
\end{abstract}

\section{What this study adds to the field}

This 10-year follow-up study demonstrated that soluble $\mathrm{P}$-selectin has prognostic values in predicting the cardiac events in patients with preserved left ventricular systolic function. This prognostic value is better than that provided by traditional biomarkers. 
the effects of blood sP-sel levels on predicting future cardiovascular events have provided controversial results. ${ }^{[3]}$

Increased levels of sP-sel have been shown to predict major cardiovascular events in patients with existing peripheral or coronary atherosclerosis.$^{[3]}$ However, a few large-scale studies were unable to demonstrate the predictive value of sP-sel on cardiovascular events. ${ }^{[4]}$ The discrepancy may be derived from different study populations without excluding the impact of left ventricular systolic function on disease outcomes. On the other hand, a few biomarkers have been investigated on their values to predict cardiovascular outcomes in patients with atherosclerotic diseases. High-sensitivity C-reactive protein (hs-CRP) and fibrinogen levels have been demonstrated to be good biomarkers of cardiovascular risks. ${ }^{[5,6]}$ Troponin-I (TnI) also has well-known prognostic and diagnostic values. ${ }^{[7]}$ However, there has never been a head-to-head study to compare the long-term prognostic values between these biomarkers.

Thus, our study specifically selected subjects hospitalized due to ischemic chest pain, treated non-invasively. The predictive value of sP-sel on future 10-year cardiovascular events was analyzed in subgroups with preserved or impaired left ventricular dysfunction. Furthermore, the prognostic value of sP-sel was also compared with other biomarkers, including hs-CRP, fibrinogen, and TnI.

\section{METHODS}

\section{Patients}

From February 1995 to August 1997, 170 patients hospitalized due to ischemic chest discomfort (suspected with coronary artery disease) were prospectively enrolled in the study. Exclusion criteria included: (1) coronary interventions performed during the hospitalization; (2) severe valvular heart disease; (3) a history of percutaneous coronary angioplasty or bypass surgery; (4) New York Heart Association functional class IV; and (5) refusal to join in this study. Before discharge (on average $5.7 \pm 2.1$ days after admission), blood samples were collected for the measurement of sP-sel, C-reactive protein (CRP), fibrinogen, creatine kinase (CK)-mass, and $\mathrm{TnI}$ in the core laboratory. Informed consent was obtained from all patients. The study was designed and carried out in accordance with the principles of the Declaration of Helsinki, and approval from the Ethics Review Board of Chang Gung Memorial Hospital was obtained.

\section{Measurements}

Plasma levels of sP-sel were assayed by enzyme-linked immunoassay (R and D Systems). Serum hs-CRP concentrations were measured with hs-immunoradiometric assay (range, $0.05-10 \mathrm{mg} / \mathrm{l}$ ), as described previously. Fi- brinogen was measured with an immunoturbidimetric assay using a Hitachi 911 analyzer with reagents and calibrators from Kamiya Biomedical (Seattle, WA, USA). The median values of sP-sel, fibrinogen, and hs-CRP were adopted as the cutoff point for further dichotic analysis. TnI was measured by chemiluminescence inmunoassays on automated analyzers (AccuTnI, Access; Beckman Coulter, Krefeld, Germany). The cutoff value of TnI, defined as the $99^{\text {th }}$ percentile of healthy participants, was $0.04 \mu \mathrm{g} / \mathrm{l}$.

\section{Patient follow-up}

For all patients, follow-up data were obtained every 6 months from hospital records, personal communication with the patients' physicians, telephone interviews, and patients' visits to staff physicians at regular intervals in the outpatient clinic. "Hard" outcome events were cardiac death and non-fatal myocardial infarction (MI), while "all events" included hard events and unstable angina. Cardiac death was defined as death with documentation of significant arrhythmia or cardiac arrest, or both, or death attributable to congestive heart failure or MI in the absence of any other precipitating factors. For patients who died outside of the hospital and did not receive an autopsy, sudden unexpected death was attributed to a cardiac cause. The recurrence of acute MI was defined using the standard criteria of history, ECG, and cardiac enzyme levels. Unstable angina was defined as accelerating anginal symptoms requiring hospital readmission, or progression of symptoms requiring revascularization. Because the decision for bypass surgery and coronary angioplasty might be subjective, the outcomes of patients with revascularization were excluded from the final data analysis. Only the most severe outcome was considered an end-point during the follow-up period after patients with planned revascularization were excluded.

\section{Statistical analysis}

Results are expressed as mean \pm SD for continuous variables and as percentages for categorical variables. Baseline variables were compared between groups using the $\chi^{2}$ or $t$-test. The predictive values of several clinical and biomarker variables for event-free survival were evaluated by a stepwise Cox regression analysis. Hazard ratio (HR) and 95\% confidence intervals $(95 \% \mathrm{CI})$ were also calculated. The event-free survival (Kaplan-Meier) was determined, and the differences between survival curves were tested with the log-rank test. The Cox regression model was used to identify significant independent predictors of new cardiac events, including age, gender, hypertension, smoking, left ventricular ejection fraction (LVEF), sP-sel, CRP, fibrinogen, and TnI. All statistical analyses were performed using appropriate software (SPSS for Windows, Release 6.0; SPSS, 
Chicago, IL, USA). A $p<0.05$ was considered statistically significant.

\section{RESULTS}

\section{Patient characteristics}

Baseline characteristics of the 170 patients are shown in Table 1. Patients were divided into two groups according to the LVEF ( $\mathrm{LVEF}>50 \%$ vs. $\mathrm{LVEF} \leq 50 \%$ ). Plasma levels of sP-sel were higher in patients with diabetes mellitus compared to those without $(85.0 \pm 41.0 \mathrm{ng} / \mathrm{ml}$ vs. $68.9 \pm 25.8 \mathrm{ng} / \mathrm{ml}$, respectively; $p=0.009)$ and in patients of younger age $(r=0.22, p=0.01)$, but were not significantly related to other laboratory and demographic parameters. To test whether the sP-sel level had prognostic values, the median value of sP-sel $(63.5 \mathrm{ng} / \mathrm{ml})$ in the group with LVEF $>50 \%$ was used as a cutoff value to separate each group into two subgroups. In the patients with LVEF $>50 \%$, patients with an sP-sel level $\geq 63.5 \mathrm{ng} / \mathrm{ml}$ were younger and had a higher prevalence of smoking and alcohol intake, compared to the patients with an $\mathrm{sP}-\mathrm{sel}$ level $<63.5 \mathrm{ng} / \mathrm{ml}$. There were no significant differences in other parameters between these two subgroups. In the patients with $\mathrm{LVEF} \leq 50 \%$, there were no significant differences in all parameters between these two subgroups.

Among the other measures of blood parameters, TnI levels were positively correlated with fibrinogen $(p=0.01)$ and hs-CRP levels $(p=0.0002)$ and negatively related to age $(p=0.0004)$. Fibrinogen levels were positively correlated with hs-CRP $(p<0.0001)$ and TnI levels $(p=0.01)$. Moreover, hs-CRP levels were associated with higher fibrinogen levels $(p<0.0001)$ and lower LVEF $(p=0.04)$. The median values of fibrinogen $(257.9 \mathrm{mg} / \mathrm{dl})$ and hs-CRP $(2.9 \mathrm{mg} / \mathrm{l})$ were used as the cutoff values for further analysis. A TnI level of $>0.4 \mathrm{ng} / \mathrm{ml}$ was used as an elevated value for further prognostic analysis.

\section{Cardiac events during 10-year follow-up}

With a median follow-up time of $9.86 \pm 3.87$ years, of the 170 patients, 44 (25.9\%) developed unstable angina, $25(14.7 \%)$ had non-fatal MI, and $14(8.2 \%)$ had cardiac deaths. The patients with LVEF $>50 \%$, compared to those with $\mathrm{LVEF} \leq 50 \%$, had insignificant differences in the incidence of unstable angina $(29.8 \%$ vs. $21.1 \%, p=0.20)$, non-fatal MI $(14.9 \%$ vs. $14.5 \%$,

Table 1: Demographic and laboratory variables in patients with a left ventricular ejection fraction of $>50 \%$ and those with $50 \%$

\begin{tabular}{|c|c|c|c|c|c|c|}
\hline \multirow[t]{2}{*}{ Variables } & \multicolumn{2}{|c|}{ LVEF $>50 \%$} & \multirow[t]{2}{*}{$p$ value } & \multicolumn{2}{|c|}{$\mathrm{LVEF} \leq 50 \%$} & \multirow[t]{2}{*}{$p$ value } \\
\hline & $\begin{array}{c}\mathrm{sP}-\mathrm{sel} \geq 63.5 \\
\mathrm{ng} / \mathrm{ml}(n=48)\end{array}$ & $\begin{array}{c}\mathrm{sP}-\mathrm{sel}<63.5 \mathrm{ng} / \mathrm{ml} \\
(n=46)\end{array}$ & & $\begin{array}{c}\mathrm{sP}-\mathrm{sel} \geq 63.5 \mathrm{ng} / \\
\mathrm{ml}(n=42)\end{array}$ & $\begin{array}{c}\mathrm{sP}-\mathrm{sel}<63.5 \mathrm{ng} / \mathrm{ml} \\
(n=34)\end{array}$ & \\
\hline Age (years) & $60.2 \pm 8.6$ & $67.1 \pm 9.8$ & 0.01 & $66.2 \pm 10.9$ & $68.8 \pm 9.7$ & 0.29 \\
\hline Sex, $n$ (male, $\%)$ & $42(87.5)$ & $34(73.9)$ & 0.09 & $35(83.3)$ & $28(82.4)$ & 0.91 \\
\hline Diabetes mellitus, $n(\%)$ & $12(25.0)$ & $16(34.8)$ & 0.30 & $16(38.1)$ & $9(26.5)$ & 0.29 \\
\hline Hypertension, $n(\%)$ & $24(50.0)$ & $20(43.5)$ & 0.53 & $18(42.9)$ & $13(38.2)$ & 0.69 \\
\hline Hyperlipidemia, $n(\%)$ & $30(62.5)$ & $22(47.8)$ & 0.15 & $22(52.4)$ & $11(32.4)$ & 0.08 \\
\hline Smoking, $n(\%)$ & $36(75)$ & $18(39.1)$ & 0.001 & $25(59.5)$ & $19(55.9)$ & 0.75 \\
\hline Alcohol intake, $n(\%)$ & $18(37.5)$ & $6(13.0)$ & 0.007 & $10(23.8)$ & $11(32.4)$ & 0.41 \\
\hline $\operatorname{LVEF}(\%)$ & $61.3 \pm 7.1$ & $60.9 \pm 7.4$ & 0.84 & $37.6 \pm 10.9$ & $35.8 \pm 11.0$ & 0.48 \\
\hline $\mathrm{CRP}(\mathrm{mg} / \mathrm{l})$ & $11.7 \pm 22.5$ & $3.8 \pm 7.3$ & 0.12 & $10.0 \pm 17.7$ & $15.1 \pm 25.7$ & 0.31 \\
\hline Fibrinogen (mg/dl) & $256.9 \pm 91.6$ & $249.3 \pm 66.8$ & 0.75 & $262.0 \pm 78.4$ & $274.8 \pm 77.7$ & 0.48 \\
\hline Troponin-I (ng/ml) & $4.9 \pm 8.6$ & $2.0 \pm 5.6$ & 0.18 & $1.3 \pm 2.5$ & $1.4 \pm 1.9$ & 0.91 \\
\hline
\end{tabular}

Abbreviations: LVEF: Left ventricular ejection fraction; CRP: C-reactive protein; sP-sel: Soluble P-selectin

Table 2: Cardiac events during the 10 years of follow-up in different patients groups

\begin{tabular}{|c|c|c|c|c|c|c|c|c|c|}
\hline & \multicolumn{2}{|c|}{ LVEF $>50 \%$} & \multirow[t]{2}{*}{$p$ value } & \multicolumn{2}{|c|}{ LVEF $50 \%$} & \multirow[t]{2}{*}{$p$ value } & \multicolumn{2}{|c|}{ All patients } & \multirow[t]{2}{*}{$p$ value } \\
\hline & $\begin{array}{c}\mathrm{sP}-\mathrm{sel} \geq 63.5 \\
\mathrm{ng} / \mathrm{ml}(n=48)\end{array}$ & $\begin{array}{c}\text { sP-sel<63.5 } \\
\mathrm{ng} / \mathrm{ml}(n=46)\end{array}$ & & $\begin{array}{c}\mathrm{sP}-\mathrm{sel} \geq 63.5 \\
\mathrm{ng} / \mathrm{ml}(n=42)\end{array}$ & $\begin{array}{c}\mathrm{sP}-\mathrm{sel}<63.5 \\
\mathrm{ng} / \mathrm{ml}(n=34)\end{array}$ & & $\begin{array}{c}\mathrm{sP}-\mathrm{sel} \leq 63.5 \\
\mathrm{ng} / \mathrm{ml}(n=90)\end{array}$ & $\begin{array}{c}\text { sP-sel<63.5 } \\
\mathrm{ng} / \mathrm{ml}(n=80)\end{array}$ & \\
\hline $\mathrm{UA}, n(\%)$ & $18(38)$ & $10(22)$ & 0.10 & $9(21)$ & $7(21)$ & 0.93 & $27(30)$ & $17(21)$ & 0.19 \\
\hline AMI, $n(\%)$ & $10(21)$ & $4(9)$ & 0.10 & $7(17)$ & $4(12)$ & 0.75 & $17(19)$ & $8(10)$ & 0.10 \\
\hline Death, $n(\%)$ & $4(8)$ & $2(4)$ & 0.68 & $4(10)$ & $4(12)$ & 1.0 & $8(9)$ & $6(8)$ & 0.74 \\
\hline UA or AMI, $n(\%)$ & $20(42)$ & $8(17)$ & 0.01 & $11(26)$ & $8(24)$ & 1.0 & $31(34)$ & $16(20)$ & 0.04 \\
\hline AMI or death, $n(\%)$ & $12(25)$ & $4(9)$ & 0.04 & $10(24)$ & $7(21)$ & 0.74 & $22(24)$ & $11(14)$ & 0.08 \\
\hline All events, $n(\%)$ & $24(50)$ & $10(22)$ & 0.004 & $15(36)$ & $12(35)$ & 1.0 & $39(43)$ & $22(28)$ & 0.03 \\
\hline
\end{tabular}

Abbreviations: LVEF: Left ventricular ejection fraction; UA: Unstable angina; AMI: Non-fatal acute myocardial infarction; all, UA or AMI or death, Statistical analysis was performed by $\chi^{2}$; sP-sel: Soluble P-selectin 
$p=0.94)$, and cardiac death $(6.4 \%$ vs. $10.5 \%, p=0.33)$. Table 2 shows that in patients with an LVEF $>50 \%$, an sP-sel level of $\geq 63.5 \mathrm{ng} / \mathrm{ml}$ was significantly associated with a higher incidence of "unstable angina and non-fatal MI" $(p=0.01)$, "hard events" $(p=0.04)$, and "all events" ( $p=0.004)$, but was not significantly associated with individual event categories (by $\chi^{2}$ analysis). However, in patients with an LVEF $\leq 50 \%$, sP-sel levels were not related to any of the cardiac or composite events. In the following analysis, prognostic values were analyzed only on "all" cardiac events.

\section{COX univariable analysis}

Univariable analysis showed that in the patients with an LVEF > 50\%, an sP-sel level of $\geq 63.5 \mathrm{ng} / \mathrm{ml}$ significantly predicted the development of all cardiac events $(p=0.04)$ [Table 3]. Figure 1 demonstrates that the curve of sP-sel level $\geq 63.5 \mathrm{ng} / \mathrm{ml}$ continuously separated from the curve of sP-sel level $<63.5 \mathrm{ng} / \mathrm{ml}$ during the 10 -year follow-up period. However, sP-sel level did not have significantly predictive values on cardiac events in patients with an LVEF $\leq 50 \%$ or in all patients [Tables 4 and 5, Figure 1].

In the patients with an LVEF $\leq 50 \%$, although the patients with a higher TnI level had a higher cardiac event rate in the first year of follow-up ( $p=0.026)$, there was no statistical significance in the long run [Table 4, Figure 2]. In the patients with an LVEF $>50 \%$, if the TnI level was elevated, there was an insignificant trend to have a higher event rate for those with follow-up longer than 5 years [Table 3, Figure 2]. The levels of fibrinogen and hs-CRP, and all demographic parameters did not provide a significant value in predicting cardiac outcomes in all patients, or in the patients with an LVEF $>50 \%$ or $\leq 50 \%$ [Tables 3-5, Figures 1 and 2].

\section{COX multivariable analysis}

Multivariable analysis by the Cox regression model demonstrated that an $\mathrm{sP}$-sel level of $>63.5 \mathrm{ng} / \mathrm{ml}$ was an independent parameter in predicting future "all" composite cardiac events only in patients with an LVEF $>50 \%(p=0.02)$, with an HR of $5.86(95 \% \mathrm{CI}=1.23-27.86)$. However, in all

Table 3: Univariable and multivariable analysis on long-term cardiac outcomes in patients with left ventricular ejection fraction $>50 \%$

\begin{tabular}{|c|c|c|c|c|}
\hline & \multicolumn{2}{|c|}{ Univariable } & \multicolumn{2}{|c|}{ Multivariable } \\
\hline & Hazard ratio $(95 \% \mathrm{CI})$ & $p$ value & Hazard ratio $(95 \% \mathrm{CI})$ & $p$ value \\
\hline Age $\geq 67$ years & $0.96(0.37-2.50)$ & 0.94 & $2.79(0.63-12.29)$ & 0.18 \\
\hline Male gender & $0.60(0.21-1.70)$ & 0.34 & $0.35(0.07-1.86)$ & 0.22 \\
\hline Diabetes mellitus & $1.00(0.35-2.84)$ & 0.99 & $0.86(0.21-3.55)$ & 0.84 \\
\hline Hypertension & $0.52(0.19-1.41)$ & 0.20 & $0.29(0.08-1.06)$ & 0.06 \\
\hline Hyperlipidemia & $0.98(0.38-2.55)$ & 0.97 & $0.93(0.28-3.04)$ & 0.90 \\
\hline Smoking & $0.83(0.32-2.16)$ & 0.71 & $1.13(0.20-6.49)$ & 0.89 \\
\hline Alcohol intake & $0.93(0.30-2.85)$ & 0.90 & $0.65(0.15-2.80)$ & 0.56 \\
\hline Troponin- $\mathrm{I}>0.4 \mathrm{ng} / \mathrm{ml}$ & $1.64(0.62-4.31)$ & 0.32 & $1.98(0.57-6.91)$ & 0.29 \\
\hline Fibrinogen $\geq 257.9 \mathrm{mg} / \mathrm{dl}$ & $1.40(0.53-3.68)$ & 0.50 & $0.49(0.12-2.07)$ & 0.34 \\
\hline $\mathrm{CRP} \geq 2.9 \mathrm{mg} / \mathrm{l}$ & $0.89(0.34-2.30)$ & 0.80 & $0.61(0.20-1.94)$ & 0.41 \\
\hline sP-selectin $\geq 63.5 \mathrm{ng} / \mathrm{ml}$ & $2.85(1.00-8.10)$ & 0.04 & $8.42(1.65-43.05)$ & 0.01 \\
\hline
\end{tabular}

Abbreviations: CRP: C-reactive protein; CI: Confidence interval; sP-sel: Soluble P-selectin

Table 4: Univariable and multivariable analysis on long-term cardiac outcomes in patients with left ventricular ejection fraction $\leq 50 \%$

\begin{tabular}{|c|c|c|c|c|}
\hline & \multicolumn{2}{|c|}{ Univariable } & \multicolumn{2}{|c|}{ Multivariable } \\
\hline & Hazard ratio $(95 \% \mathrm{CI})$ & $p$ value & Hazard ratio $(95 \% \mathrm{CI})$ & $p$ value \\
\hline Age $\geq 67$ years & $0.77(0.36-1.63)$ & 0.49 & $0.90(0.35-2.27)$ & 0.82 \\
\hline Male gender & $0.92(0.35-2.44)$ & 0.87 & $0.68(0.22-2.12)$ & 0.50 \\
\hline Diabetes mellitus & $1.34(0.61-2.92)$ & 0.47 & $1.32(0.59-2.96)$ & 0.50 \\
\hline Hypertension & $1.77(0.83-3.77)$ & 0.14 & $1.74(0.78-3.91)$ & 0.18 \\
\hline Hyperlipidemia & $1.40(0.66-2.97)$ & 0.39 & $1.05(0.43-2.59)$ & 0.91 \\
\hline Smoking & $1.58(0.71-3.53)$ & 0.26 & $1.35(0.47-3.90)$ & 0.58 \\
\hline Alcohol intake & $1.67(0.77-3.66)$ & 0.20 & $1.35(0.44-4.09)$ & 0.60 \\
\hline Troponin-I $>0.4 \mathrm{ng} / \mathrm{ml}$ & $1.26(0.57-2.74)$ & 0.57 & $1.32(0.54-3.25)$ & 0.54 \\
\hline Fibrinogen $257.9 \mathrm{mg} / \mathrm{dl}$ & $1.10(0.52-2.35)$ & 0.81 & $0.95(0.36-2.50)$ & 0.92 \\
\hline $\mathrm{CRP} \geq 2.9 \mathrm{mg} / \mathrm{l}$ & $0.93(0.43-1.99)$ & 0.84 & $0.96(0.39-2.35)$ & 0.93 \\
\hline $\mathrm{sP}$-selectin $\geq 63.5 \mathrm{ng} / \mathrm{ml}$ & $1.07(0.50-2.30)$ & 0.85 & $1.06(0.45-2.49)$ & 0.90 \\
\hline
\end{tabular}

Abbreviations: CRP: C-reactive protein; CI: Confidence interval; sP-sel: Soluble P-selectin 


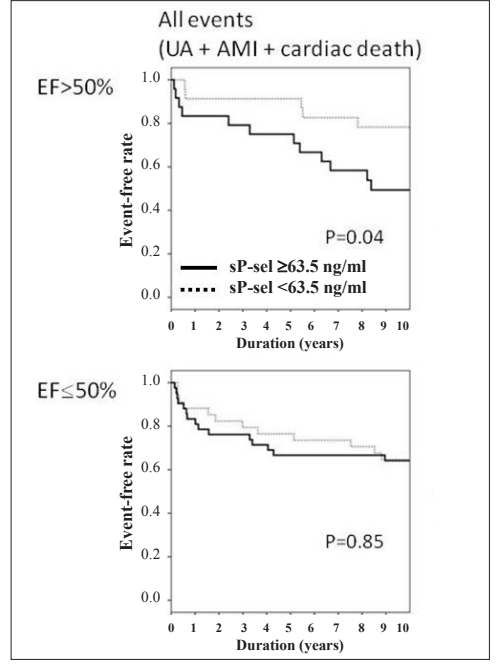

Figure 1: The 10-year prognostic values of soluble P-selectin (sP-sel) levels on "all" cardiac events in patients with a left ventricular ejection fraction $>50 \%$ and $\leq 50 \%$ (UA, unstable angina; AMI, non-fatal acute myocardial infarction) patients or in patients with an LVEF $\leq 50 \%$, there were no demographic or laboratory parameters significantly associated with the development of cardiac events.

\section{DISCUSSION}

In patients with ischemic chest pain and treated with non-invasive strategy, our data showed that plasma sP-sel level is a good predictor of cardiac events during the 10-year follow-up period in the population with an LVEF $>50 \%$. Clinical parameters, hs-CRP, fibrinogen, and TnI have no significant prognostic values in this population. However, the prognostic value of sP-sel could not be demonstrated in the patients with an LVEF $\geq 50 \%$.

\section{Previous studies}

Blood sP-sel concentrations are increased in a variety of thrombotic and atherosclerotic diseases in human beings. ${ }^{[8]}$

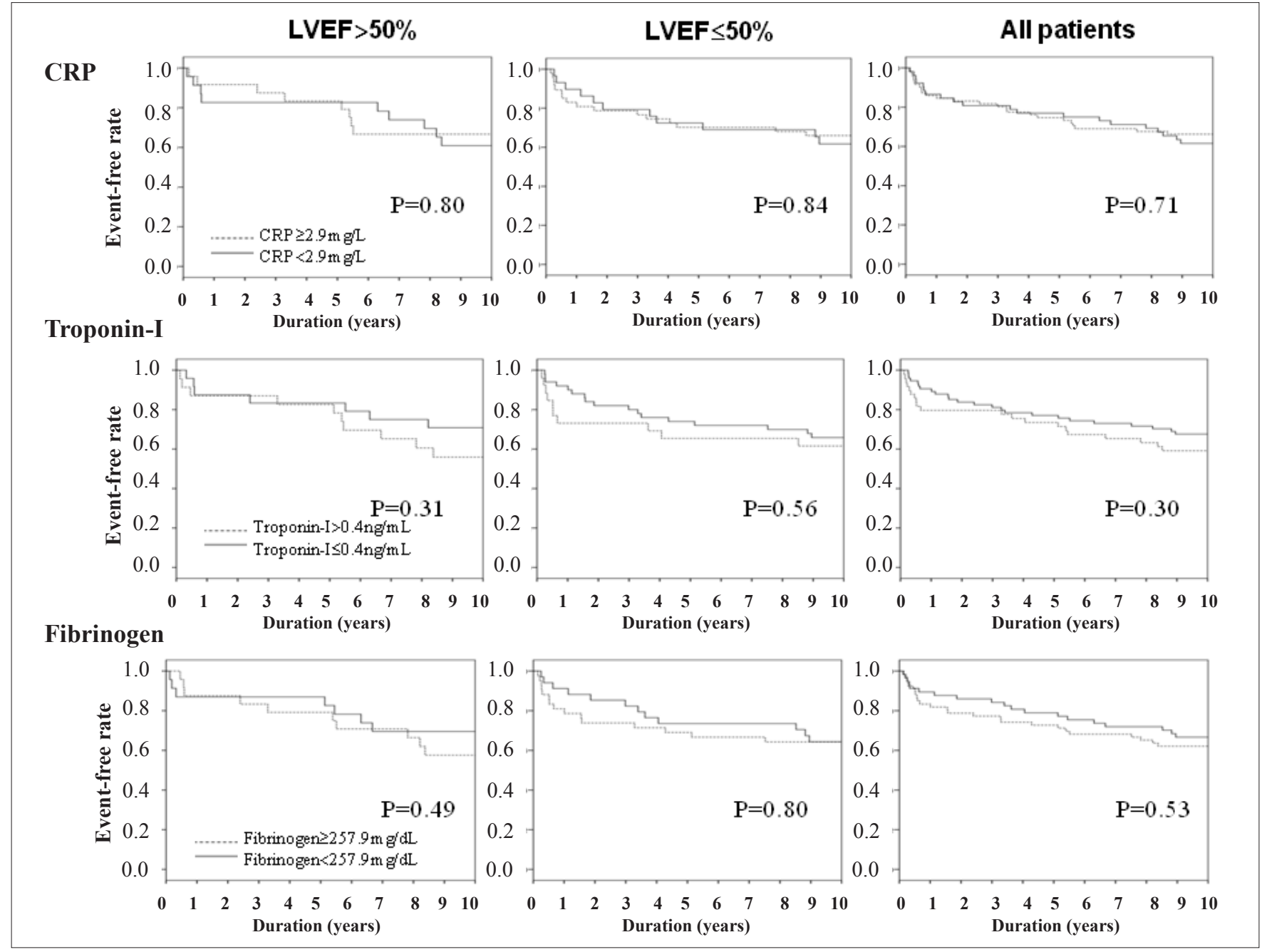

Figure 2: The 10-year prognostic values of high-sensitivity C-reactive protein (hs-CRP), troponin I, and fibrinogen levels on "all” cardiac events in patients with a left ventricular ejection fraction $>50 \%$ and $\leq 50 \%$ 
Table 5: Univariable and multivariable analysis on long-term cardiac outcomes in all patients

\begin{tabular}{|c|c|c|c|c|}
\hline & \multicolumn{2}{|c|}{ Univariable } & \multicolumn{2}{|c|}{ Multivariable } \\
\hline & Hazard ratio $(95 \% \mathrm{CI})$ & $p$ value & Hazard ratio $(95 \% \mathrm{CI})$ & $p$ value \\
\hline Age $\geq 67$ years & $0.86(0.47-1.55)$ & 0.61 & $1.13(0.56-2.27)$ & 0.73 \\
\hline Male gender & $0.78(0.38-1.58)$ & 0.49 & $0.59(0.26-1.36)$ & 0.22 \\
\hline Diabetes mellitus & $1.22(0.66-2.28)$ & 0.53 & $1.12(0.58-2.15)$ & 0.74 \\
\hline Hypertension & $1.13(0.63-2.05)$ & 0.68 & $1.02(0.55-1.92)$ & 0.94 \\
\hline Hyperlipidemia & $1.21(0.67-2.18)$ & 0.53 & $1.01(0.53-1.91)$ & 0.98 \\
\hline Smoking & $1.23(0.67-2.26)$ & 0.51 & $1.18(0.52-2.71)$ & 0.70 \\
\hline Alcohol intake & $1.37(0.73-2.58)$ & 0.33 & $1.40(0.63-3.09)$ & 0.41 \\
\hline Troponin-I $>0.4 \mathrm{ng} / \mathrm{ml}$ & $1.36(0.75-2.47)$ & 0.31 & $1.45(0.74-2.87)$ & 0.28 \\
\hline Fibrinogen $\geq 257.9 \mathrm{mg} / \mathrm{dl}$ & $1.21(0.67-2.19)$ & 0.54 & $1.09(0.56-2.11)$ & 0.80 \\
\hline $\mathrm{CRP} \geq 2.9 \mathrm{mg} / \mathrm{l}$ & $0.89(0.49-1.62)$ & 0.71 & $0.87(0.46-1.62)$ & 0.65 \\
\hline sP-selectin $\geq 63.5 \mathrm{ng} / \mathrm{ml}$ & $1.53(0.83-2.80)$ & 0.17 & $1.48(0.78-2.81)$ & 0.24 \\
\hline
\end{tabular}

Abbreviations: CRP: C-reactive protein; CI: Confidence interval; sP-sel: Soluble P-selectin

However, studies on the effects of sP-sel plasma levels on predicting future cardiovascular events have provided controversial results. Mulvihill et al. found that raised serum sP-sel levels were unable to predict cardiovascular events in a small population with unstable angina and MI. ${ }^{[9]}$ Malik et al., performing a 16-year follow-up of 643 men with coronary artery disease and 1278 controls, found that serum sP-sel was unlikely to add much predictive information to established risk factors. ${ }^{[4]}$ However, in population-based studies, increased levels of sP-sel in citrated plasma have been shown to predict major cardiovascular events in patients with existing peripheral or coronary atherosclerosis and in apparently healthy women. ${ }^{[10]}$ The discrepancy may be derived from different blood preparation and different study designs, without considering the impact of left ventricular systolic function on disease outcomes. Our study separately looked at the prognostic value of plasma sP-sel levels in patients with preserved and impaired left ventricular systolic function.

\section{Findings in our study}

sP-sel was a valuable predictor of future cardiac events only in the patients with an LVEF $>50 \%$. Actually, the major prognostic role of sP-sel is the prediction of recurrent unstable angina and non-fatal acute MI, with a minor role on the prediction of cardiac death. Usually, in patients with preserved left ventricular function, thrombus-associated coronary instability and acute closure are the major causes of coronary events. Interestingly, our data revealed that the plasma concentration of sP-sel continuously provided the prognostic information from the time point of patients enrolled throughout the whole period of 10 years. This finding suggests that patients with elevated sP-sel levels had a trait of re-activation of coronary instability.

It is surprising that $\mathrm{sP}-\mathrm{sel}$ levels did not have prognostic values in patients with left ventricular dysfunction. Patients with heart failure exhibit evidence of abnormal platelet activation. ${ }^{[11]}$ Circulating levels of adhesion molecules, including sP-sel, have been found to be elevated in patients with heart failure compared with controls. ${ }^{[12]}$ Furthermore, no differences in platelet parameters could be demonstrated when ischemic and non-ischemic etiologies of heart failure were compared. ${ }^{[13]}$ The generally increased sP-sel levels in heart failure patients may mask the prognostic value of $\mathrm{sP}-\mathrm{sel}$ on future ischemic events. Keeping in line with this notion, Chung et al. demonstrated that p-selectin lacked prognostic value in patients with heart failure. ${ }^{[14]}$ None of a variety of platelet markers was predictive of the composite end-point at follow-up. These findings further support that $\mathrm{sP}$-sel provides prognostic values only in patients without interference by heart failure.

\section{Prognostic value of hs-CRP, fibrinogen, and TnI}

Hs-CRP is a well-accepted marker of inflammation. In studies involving large numbers of patients, hs-CRP levels seem to be correlated with the levels of cardiovascular risk. ${ }^{[15]}$ In fact, hs-CRP seems to predict cardiovascular risk at least as well as cholesterol levels do. ${ }^{[16]}$ Data from the Physicians' Health Study, a clinical trial involving 18,000 apparently healthy physicians, revealed that elevated levels of hs-CRP were associated with a threefold increase in the risk of heart attack. ${ }^{[17]}$ However, in our study, the patients enrolled were suggested to be at a relatively higher risk than those in the community-based clinical trials, as indicated by the hospitalization due to ischemic chest pain and a high prevalence of increased hs-CRP level. Accordingly, the prognostic value of hs-CRP in this population may be attenuated. On the other hand, the role of fibrinogen in the development of cardiovascular disease has been fully confirmed by the results of all relevant studies conducted during the past 10 years. ${ }^{[6]}$ Figure 2 shows that there is only a trend of relationship between higher fibrinogen levels and a poor cardiac outcome in patients with an LVEF $>50 \%$. Small 
patient population in our study may also limit the demonstration of both hs-CRP and fibrinogen on prognostic values.

Troponin assays are not only more sensitive but are also more specific than CK-MB assays. ${ }^{[18]}$ Although most previous studies showed that even minor elevations in troponin levels presage short- and long-term events ${ }^{[19]}$ the prognostic value of TnI was only demonstrated in the first year follow-up in our patients with impaired cardiac systolic function. TnI level was not as good as sP-sel in our study designed by a head-to-head comparison for 10-year long-term follow-up. However, Figure 2 demonstrates that there is a trend that a higher TnI level is associated with a higher cardiac event rate if the follow-up is longer than 5 years. A longer follow-up may delineate a new aspect of $\mathrm{TnI}$ levels on prognostic values.

\section{Study limitations}

There are a few limitations in this study. First, the cutoff value for each parameter should be better estimated by receiver operating characteristic curves. However, limited by small sample size in this 10-year survival analysis, only median values were used to ensure adequate numbers in each subgroup and reach the goal of head-to-head comparisons. Second, guideline-based medications have a significant impact on the cardiovascular outcomes. However, without an intention-to-treatment program, this study designed 10 years ago could not provide conclusive information on this point. Third, incorporating the data of kidney function and factors interfering with the levels of biomarkers would strengthen the independent predictive values of these biomarkers. Finally, the clinical application of the findings in our study is limited in the population described in our enrollment criteria. Further studies designed based on these limitations are definitely necessary.

\section{CONCLUSION}

Plasma sP-sel provides useful prognostic value on long-term cardiovascular outcomes in patients with preserved left ventricular function and suggested with coronary artery disease. This prognostic value is even better than that provided by traditionally well-documented biomarkers, including hs-CRP, fibrinogen, and TnI.

\section{REFERENCES}

1. Koedam JA, Cramer EM, Briend E, Furie B, Furie BC, Wagner DD. $\mathrm{P}$-selectin, a granule membrane protein of platelets and endothelial cells, follows the regulated secretory pathway in AtT-20 cells. J Cell Biol 1992;116:617-25.

2. De PG, Pannacciulli N, Coviello M, Scarangella A, Di RP, Caringella M, et al. sP-selectin plasma levels in obesity: Association with insulin resistance and related metabolic and prothrombotic factors. Nutr Metab Cardiovasc Dis 2008;18:227-32.
3. Blann AD, Nadar SK, Lip GY. The adhesion molecule P-selectin and cardiovascular disease. Eur Heart J 2003;24:2166-79.

4. Malik I, Danesh J, Whincup P, Bhatia V, Papacosta O, Walker M, et al. Soluble adhesion molecules and prediction of coronary heart disease: A prospective study and meta-analysis. Lancet 2001;358:971-6.

5. Wilson PW, Pencina M, Jacques P, Selhub J, D'Agostino RSr, O'Donnell CJ. C-reactive protein and reclassification of cardiovascular risk in the Framingham Heart Study. Circ Cardiovasc Qual Outcomes 2008;1:92-7.

6. Ernst E, Resch KL. Fibrinogen as a cardiovascular risk factor: A meta-analysis and review of the literature. Ann Intern Med 1993;118:956-63.

7. Antman EM, Tanasijevic MJ, Thompson B, Schactman M, McCabe CH, Cannon CP, et al. Cardiac-specific troponin I levels to predict the risk of mortality in patients with acute coronary syndromes. N Engl J Med 1996;335:1342-9.

8. Blann AD, Lip GY. Cell adhesion molecules in cardiovascular disease and its risk factors: What can soluble levels tell us? J Clin Endocrinol Metab 2000;85:1745-7.

9. Mulvihill NT, Foley JB, Murphy RT, Curtin R, Crean PA, Walsh M. Risk stratification in unstable angina and non-Q wave myocardial infarction using soluble cell adhesion molecules. Heart 2001;85:623-7.

10. Merten M, Thiagarajan P. P-selectin in arterial thrombosis. Z Kardiol 2004;93:855-63

11. Chung I, Choudhury A, Patel J, Lip GY. Soluble, platelet-bound, and total P-selectin as indices of platelet activation in congestive heart failure. Ann Med 2009;41:45-51.

12. Andreassen AK, Nordoy I, Simonsen S, Ueland T, Muller F, Froland SS, et al. Levels of circulating adhesion molecules in congestive heart failure and after heart transplantation. Am J Cardiol $1998 ; 81: 604-8$

13. Gurbel PA, Gattis WA, Fuzaylov SF, Gaulden L, Hasselblad V, Serebruany VL, et al. Evaluation of platelets in heart failure: Is platelet activity related to etiology, functional class, or clinical outcomes? Am Heart J 2002; 143:1068-75.

14. Chung I, Choudhury A, Lip GY. Platelet adhesion in congestive heart failure. Thromb Res 2008;121:885-8.

15. Lagrand WK, Visser CA, Hermens WT, Niessen HW, Verheugt FW, Wolbink GJ, et al. C-reactive protein as a cardiovascular risk factor: More than an epiphenomenon? Circulation 1999;100:96-102.

16. Ridker PM, Rifai N, Rose L, Buring JE, Cook NR. Comparison of $\mathrm{C}$-reactive protein and low-density lipoprotein cholesterol levels in the prediction of first cardiovascular events. N Engl J Med 2002;347:1557-65

17. Belanger C, Buring JE, Cook N, Eberlein K, Goldhaber SZ, Gordon D. Final report on the aspirin component of the ongoing Physicians' health study. Steering committee of the physicians' health study research group. N Engl J Med 1989;321:129-35.

18. Wu AH, Feng YJ, Contois JH, Pervaiz S. Comparison of myoglobin, creatine kinase-MB, and cardiac troponin I for diagnosis of acute myocardial infarction. Ann Clin Lab Sci 1996;26:291-300.

19. Pham MX, Whooley MA, Evans GTJr, Liu C, Emadi H, Tong W, et al. Prognostic value of low-level cardiac troponin-I elevations in patients without definite acute coronary syndromes. Am Heart J 2004; 148:776-82. 